\title{
Kremen1 and Dickkopf1 control cell survival in a Wnt-independent manner
}

\author{
F Causeret ${ }^{\star, 1}$, I Sumia $^{1}$ and A Pierani ${ }^{*, 1}$
}

In multicellular organisms, a tight control of cell death is required to ensure normal development and tissue homeostasis. Improper function of apoptotic or survival pathways can not only affect developmental programs but also favor cancer progression. Here we describe a novel apoptotic signaling pathway involving the transmembrane receptor Kremen1 and its ligand, the Wnt-antagonist Dickkopf1. Using a whole embryo culture system, we first show that Dickkopf1 treatment promotes cell survival in a mouse model exhibiting increased apoptosis in the developing neural plate. Remarkably, this effect was not recapitulated by chemical Wnt inhibition. We then show that Dickkopf1 receptor Kremen1 is a bona fide dependence receptor, triggering cell death unless bound to its ligand. We performed Wnt-activity assays to demonstrate that the pro-apoptotic and anti-Wnt functions mediated by Kremen1 are strictly independent. Furthermore, we combined phylogenetic and mutagenesis approaches to identify a specific motif in the cytoplasmic tail of Kremen1, which is (i) specifically conserved in the lineage of placental mammals and (ii) strictly required for apoptosis induction. Finally, we show that somatic mutations of kremen1 found in human cancers can affect its pro-apoptotic activity, supporting a tumor suppressor function. Our findings thus reveal a new Wnt-independent function for Kremen1 and Dickkopf1 in the regulation of cell survival with potential implications in cancer therapies.

Cell Death and Differentiation (2016) 23, 323-332; doi:10.1038/cdd.2015.100; published online 24 July 2015

In multicellular organisms, long-distance communication between cells is typically achieved by secreted ligands that diffuse through the extracellular medium and bind transmembrane receptors on target cells. Signal propagation through the plasma membrane is then achieved by receptor conformational changes upon ligand binding and classically involves modulation of enzymatic activity, interaction with intracellular partners or ion permeability. The classical view that transmembrane receptors only signal when bound to their ligand is now outdated, especially since the emergence of the dependence receptor concept. Dependence receptors do not form a family per se, but rather regroup a variety of receptors (transmembrane but also nuclear) that share the ability to trigger cell death unless bound to their respective ligand(s). ${ }^{1}$ Dependence receptors therefore display two signaling activities: a 'positive' signaling in the presence of a ligand that can modulate various cellular processes (proliferation, differentiation, migration, etc.), and a 'negative' signaling in the absence of ligand consisting in the activation of a pro-apoptotic cascade downstream the receptor. To date, more than a dozen dependence receptors have been identified among which, deleted in colorectal cancer (DCC), Unc5A-D, Patched, TrkA/C, PlexinD1 and others, ${ }^{1-7}$ all of which play major roles during embryonic development, especially in the nervous system. Dependence receptors are also involved in cancer: as their apoptotic activity may allow to restrain cells within a ligand-rich environment and eliminate those that migrate away (such as metastatic cells), they were proposed to act as conditional tumor suppressors. ${ }^{1}$ Accordingly, loss of function of a dependence receptor or overexpression of a ligand can confer a selective advantage to tumor cells.

Many signaling pathways involved during embryonic development are misregulated in cancers. Among them, the wingless-related integration site (Wnt)-signaling pathway has been extensively studied in both developmental and cancer paradigms. $^{8,9}$ The Wnt1 ligand itself was first identified as a proto-oncogene ${ }^{10}$ and later shown to be required for mammalian brain patterning. ${ }^{11}$ Although Wnt signaling was reported to elicit multiple cellular responses, most of the physiological effects described so far were shown to be mediated by the socalled canonical pathway: extracellular Wnt ligands bind transmembrane receptors of the Frizzled family associated with LDL receptor-related protein (LRP) co-receptors, leading to the activation of an intracellular signaling cascade whose final effector, $\beta$-catenin, modulates the expression of target genes. ${ }^{12}$ Critical regulations of the Wnt pathway occur at the extracellular level by means of secreted antagonists. ${ }^{13}$ Dickkopf-1 (Dkk1) is one of such secreted inhibitors. It was shown to bind Kremen1/2 (Krm1/2) as well as LRP5/6 transmembrane receptors and subsequently inhibit Wnt downstream signaling. ${ }^{14-17}$ During vertebrate embryonic development, Dkk1-mediated Wht antagonism in anterior regions is strictly required for head induction. ${ }^{18,19}$ In cancers,

\footnotetext{
${ }^{1}$ Institut Jacques Monod, CNRS, UMR 7592, Université Paris Diderot, Sorbonne Paris Cité, Paris, France

*Corresponding author: F Causeret or A Pierani, Institut Jacques Monod, CNRS, UMR 7592, Université Paris Diderot, Sorbonne Paris Cité, Bâtiment Buffon $516 \mathrm{~B}, 15$ rue Hélène Brion, Paris Cedex 13, 75205, France. Tel: +33 157278128; Fax: +33 157278087; E-mail: frederic.causeret@inserm.fr or alessandra.pierani@ijm.fr Abbreviations: CNS, central nervous system; COSMIC, catalogue of somatic mutations in cancer; DCC, deleted in colorectal cancer; Dkk, Dickkopf; ECD, extracellular domain; GFP, green fluorescent protein; HA, hemagglutinin; HEK, human embryonic kidney; ICD, intracellular domain; Krm, Kringle-coding gene marking the eye and the nose; LRP, low-density lipoprotein receptor-related protein; TCGA, the cancer genome atlas; TUNEL, terminal deoxynucleotidyl transferase dUTP nick end labeling; Wnt, wingless-related integration site

Received 06.1.15; revised 16.6.15; accepted 23.6.15; Edited by H Ichijo; published online 24.7.15
} 
Dkk1 function remains a matter of debate as it was proposed to act as either a positive or a negative factor depending on the context. $^{20-22}$ Such a discrepancy could reflect a yet unknown function for Dkk1 in addition to its well-characterized Wntinhibiting activity.

In this study, we unravel a new Wnt-independent antiapoptotic function for Dkk1 and find it is mediated by its transmembrane receptor Krm1, which behaves as a dependence receptor.

\section{Results}

Dkk1 is a diffusible survival factor. Using a genetic cell ablation strategy in mice, we have previously identified a non cell-autonomous mechanism regulating cell survival during early (E8.5) embryonic mouse head development (Supplementary Figure 1a). ${ }^{23}$ We aimed at identifying the diffusible factor(s) and cognate receptor(s) that could be involved in such a process. We performed in situ hybridization for a range of secreted factors, as well as their transmembrane receptors, known to be involved in head development. Consistent with previous observations, ${ }^{19,24}$ we found that Dkk1 as well as its receptors Krm1/2 and Lrp6 are expressed in the anterior neural plate at E8.5 (Supplementary Figures 1b-f).

To test the possibility that Dkk1 regulates cell survival during forebrain development, we implemented a whole embryo culture strategy and assessed its ability to rescue apoptosis observed in our mouse model. ${ }^{23}$ E7.5 embryos were dissected and maintained for $24 \mathrm{~h}$ in culture before fixation and subsequent detection of apoptotic cells by terminal deoxynucleotidyl transferase dUTP nick end labeling (TUNEL) staining. Culture conditions allowed recapitulation of in vivo conditions, that is, extensive apoptosis in the forebrain and midbrain of ablated embryos compared with wild-type littermates (Supplementary Figure 2). We found that treatment with soluble recombinant Dkk1 decreased the number of TUNEL $^{+}$apoptotic cells observed in mutants in a dosedependent manner (Figure 1), indicating that Dkk1 acts as a survival factor for embryonic mouse neural plate.

Dkk1 was shown to act as a potent inhibitor of Wnt signaling. ${ }^{18}$ To determine whether it promotes survival in a Wnt-dependent manner, we applied endo-IWR1, a chemical inhibitor of Wnt canonical signaling, ${ }^{25}$ on ablated embryos. Unlike Dkk1 treatment, endo-IWR1 failed to recue apoptosis (Figure 1), indicating that Dkk1 anti-apoptotic function is Wnt
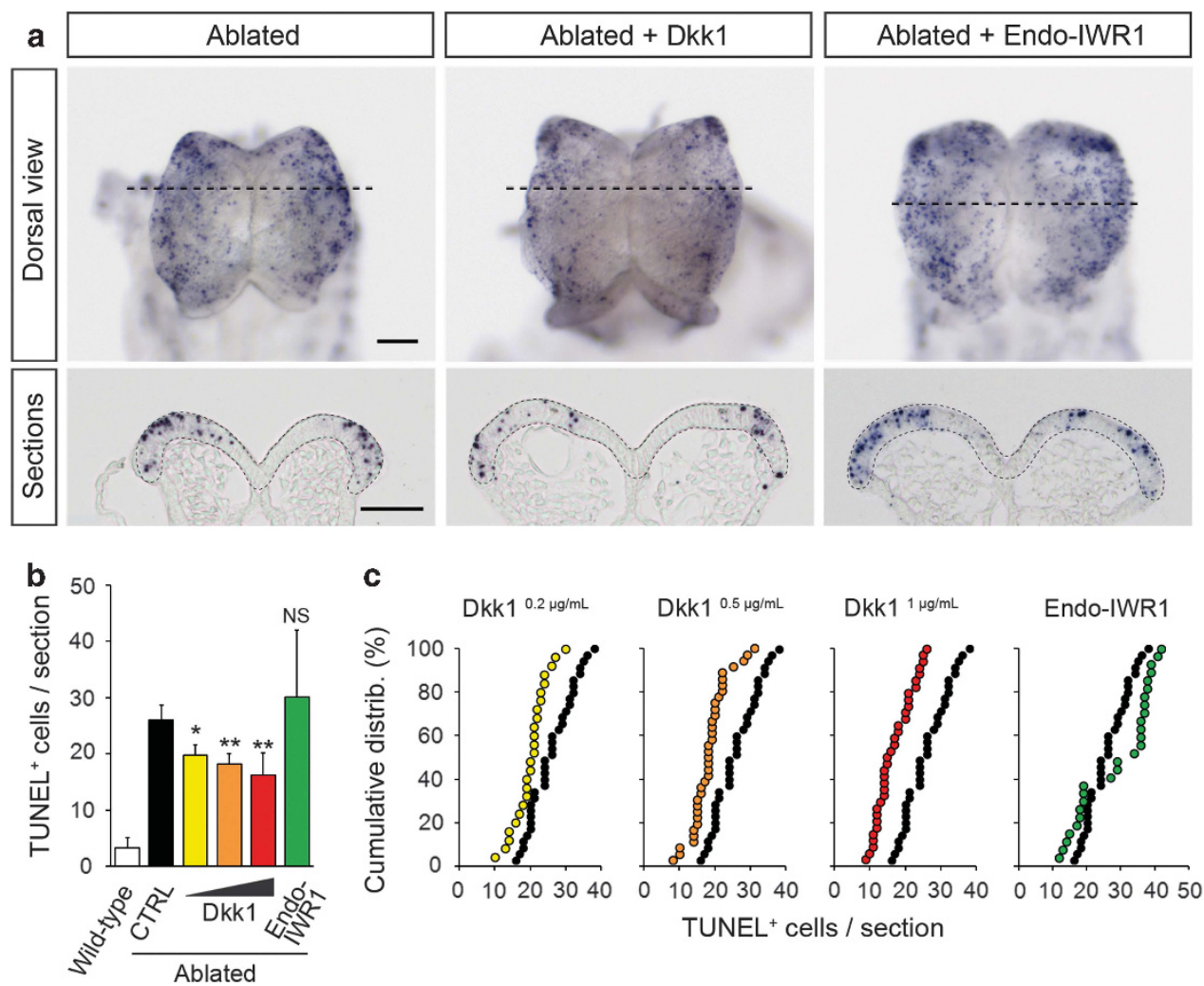

Figure 1 Dickkopf1 acts as a survival factor in a Wnt-independent manner. (a) Ablated PGK:Cre;Dbx $1^{\text {DTA }}$ embryos cultured in the absence or presence of either recombinant Dkk1 or endo-IWR1 and stained by TUNEL. Top lane is a dorsal view (anterior is up, scale bar: $100 \mu \mathrm{m}$ ). Lower lane corresponds to cryostat sections (collected at the level of the dashed line) of the embryos shown above (scale bar: $100 \mu \mathrm{m}$ ). The neural plate is surrounded by a dashed line to allow a better visualization. (b and c) Histogram (mean \pm S.D.) and cumulative distributions showing the number of TUNEL ${ }^{+}$cells per section counted in ablated embryos untreated ( 35 sections from five animals), Dkk1-treated (25 sections from three animals at $0.2 \mu \mathrm{g} / \mathrm{ml}, 36$ sections from four animals at $0.5 \mu \mathrm{g} / \mathrm{ml}$ and 34 sections from four animals at $1 \mu \mathrm{g} / \mathrm{ml}$ ) or Endo-IWR1-treated (27 sections from three animals at $10 \mu \mathrm{M}$ ); ${ }^{*} P<0.01$ using Student's $t$-test and $P<0.003$ using Kolmogorov-Smirnov test, ${ }^{* *} P<0.003$ using Student's $t$-test and $P<0.001$ using Kolmogorov-Smirnov test, NS: nonsignificant 
independent, and thus suggesting the possibility of a novel signaling pathway downstream Dkk1.

Dkk1 receptor $\mathrm{Krm} 1$ has an intrinsic pro-apoptotic activity. We then investigated the possibility that Dkk1 negatively regulates a pro-apoptotic signaling triggered by one of its receptors. Such a mechanism implies that one (or more) of Dkk1 receptors behaves as a dependence receptor. ${ }^{1}$ To assess whether Dkk1 receptors possess an intrinsic pro-apoptotic activity, we transfected HEK293T cells with plasmids encoding Krm1, Krm2 or Lrp6, and performed immunostaining against activated-Caspase-3 to reveal cells undergoing apoptosis. ${ }^{26}$ As illustrated in Figures $2 \mathrm{a}-\mathrm{d}$, Krm1, but not Krm2 or Lrp6, expression resulted in a perinuclear accumulation of activated-Caspase-3.

To better understand how the apoptotic activity of Krm1 is regulated, we generated truncation mutants lacking either the extra- or intracellular domain. We found that deletion of the intracellular domain of Krm1 $(\triangle I C D)$ completely abolished Caspase-3 activation (Figures $2 \mathrm{e}$ and f). On the contrary, a $\mathrm{Krm} 1$ construct devoid of extracellular domain $(\triangle E C D)$, despite being expressed at lower levels, appeared hyperactive as it was able to elicit strong apoptotic signaling in almost all transfected cells (Figures $2 \mathrm{~g}$ and $\mathrm{h}$ ). These observations suggest that Krm1 apoptotic activity is mediated by its ICD and negatively regulated by its $E C D$, possibly through ligand binding.

Dkk1 inhibits the pro-apoptotic activity of Krm1. As Krm1 apoptotic activity appears regulated by extracellular signals, we tested the consequences of Dkk1's presence on this process. We found that co-transfection of Dkk1 was able to inhibit Krm1-induced Caspase-3 activation (Figures 3a and b). Quantifications indicated a $\sim 40-65 \%$ decrease (depending on the Dkk1/Krm1 ratio) in the proportion of active Caspase3-positive cells among Krm1-transfected cells (Figure 3c), and the remaining apoptotic cells displayed a two- to fourfold reduction in the levels of activated Caspase- 3 fluorescence relative to hemagglutinin ( $\mathrm{HA}$ ) fluorescence (Figure $3 \mathrm{~d}$ ).

We also assessed the ability of other known Krm1 ligands Dkk2, Dkk3, Dkk4 and R-spondin1 $1^{17,27,28}$ to rescue Krm1induced apoptosis. Although none of them were found as efficient as Dkk1, all were able to decrease Caspase-3 activation upon co-transfection with $\mathrm{Krm} 1$ in a significant and dose-dependent manner (Figure 3c).

We then tested whether Dkk1 can inhibit Krm1 apoptotic activity in a non-cell autonomous manner. We first applied soluble Dkk1 (using either a recombinant protein or a conditioned medium from Dkk1-transfected cells) on Krm1transfected cells. In another set of experiments, we seeded Krm1-transfected cells on a carpet of Dkk1-expressing cells. In all cases we measured a significant decrease in the proportion of Caspase-3 positive cells among Krm1transfected cells upon exposure to exogenous Dkk1 (Figures $3 e-g$ ). The fact that we never observed as good of a rescue using nonautonomous strategies as in cotransfection experiments could be due to the protein stability/ activity issues, as previously reported, ${ }^{29}$ or to the existence of a pool of Krm1 that would remain inaccessible (intracellular for example) to exogenous ligand. From these experiments, we concluded that $\mathrm{Krm} 1$ is a bona fide dependence receptor whose apoptotic activity is inhibited upon ligand binding in a dose-dependent manner.

Krm1 and Dkk1 control cell survival in a Wntindependent manner. As Wnt inhibition in cultured embryos is unable to mimic the anti-apoptotic effect of Dkk1, we
DAPI GFP Activated Caspase-3
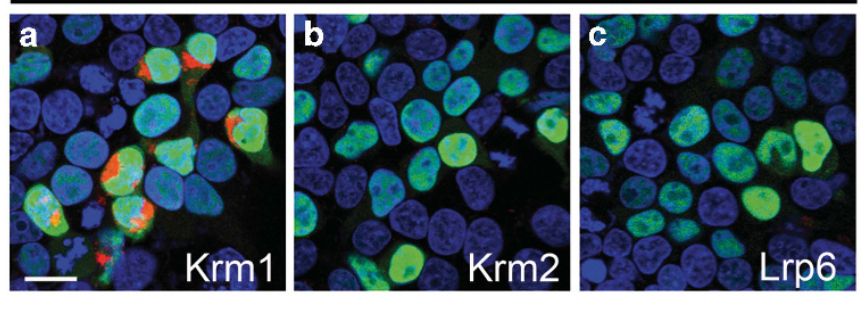

DAPI Activated Caspase-3 HA
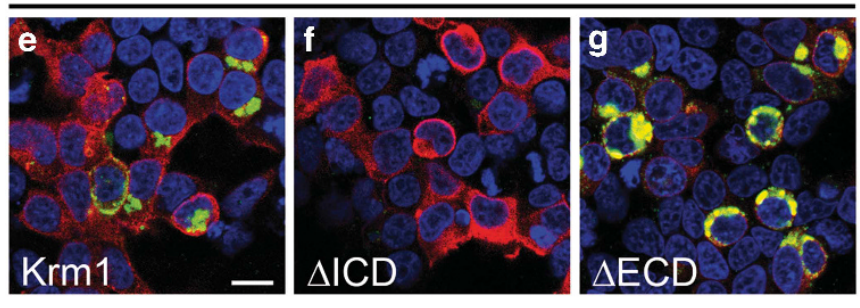
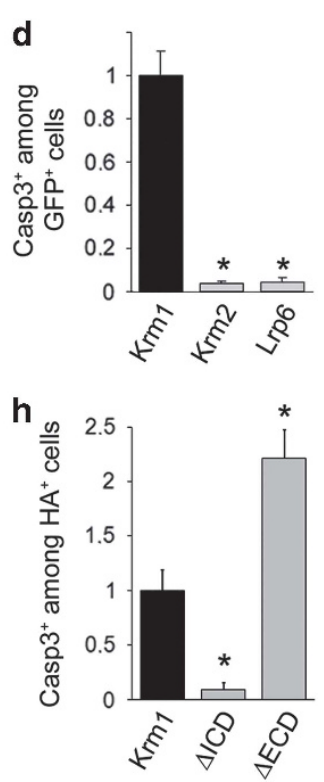

Figure 2 Kremen1 has a pro-apoptotic activity. (a-c) HEK293T cells overexpressing Krm1 (a), Krm2 (b) or Lrp6 (c) are identified by nuclear GFP expression (green). Activated Caspase-3 is revealed by immunostaining (red); scale bar: $10 \mu \mathrm{m}$. (d) Histogram representing the proportion of activated Caspase- $3^{+}$cells among GFP ${ }^{+}$cells in the experiments shown in (a-c) and normalized to 1 (mean \pm S.D.); ${ }^{*} P<0.001$ using Student's -test. (e-g) HEK293T cells transfected with HA-tagged Krm1 full length (e), lacking its intracellular domain (f) or lacking its extracellular domain (g). Cells were immunostained for HA (red) and activated Caspase-3 (green); scale bar: $10 \mu \mathrm{m}$. (h) Quantification of the proportion of activated Caspase- $3^{+}$cells among $\mathrm{HA}^{+}$cells normalized to 1 (mean \pm S.D.) in the experiments shown in $(\mathbf{f}-\mathbf{h}) ;{ }^{*} P<0.001$ using Student's $t$-test 
DAPI Activated Caspase-3 HA
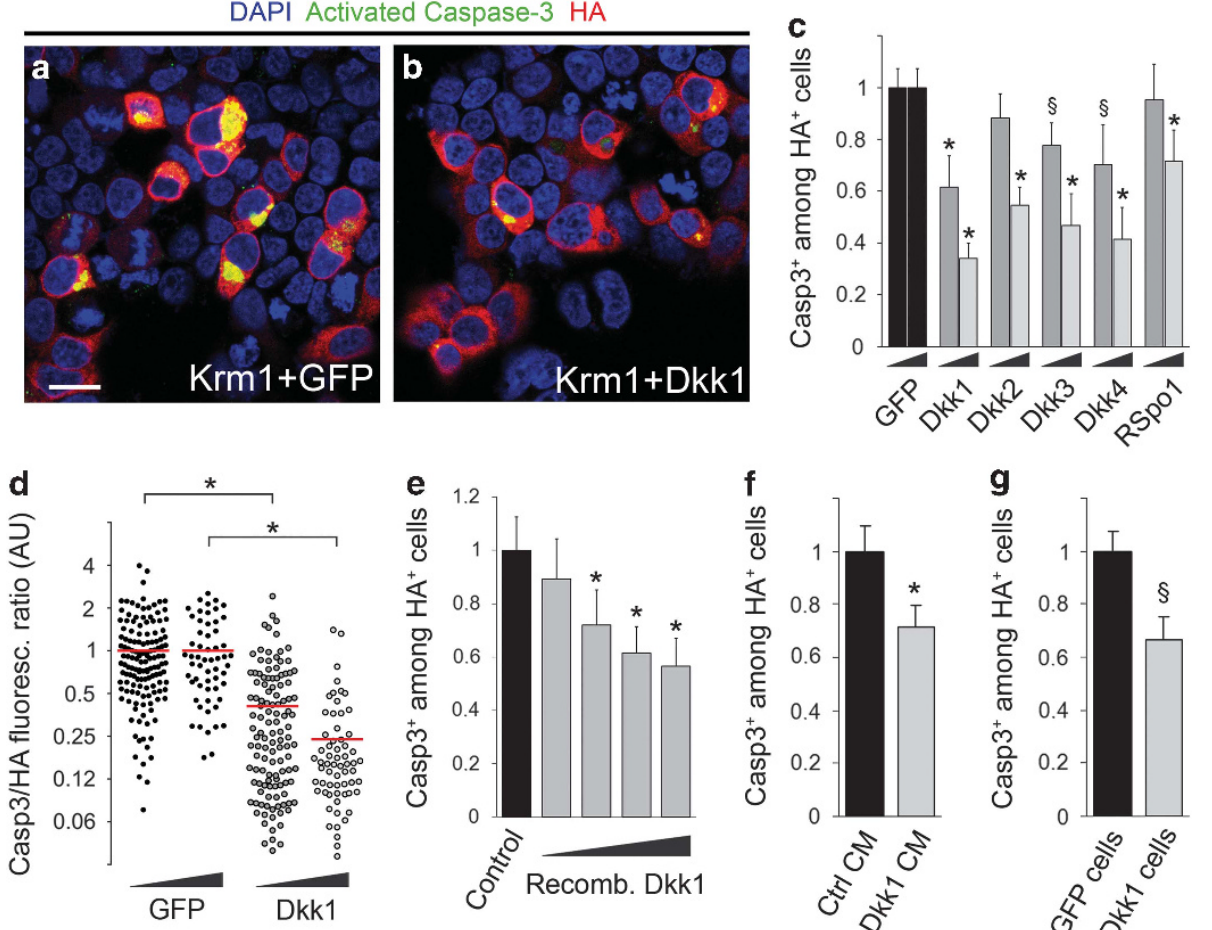

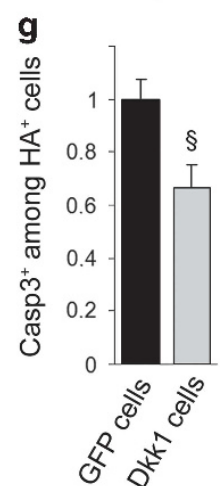

Figure 3 Kremen1 is a dependence receptor for Dickkopf1 (a and b) HEK293T cells co-transfected with HA-tagged Krm1 (red) and either GFP (a) or Dkk1 (b) and immunostained for activated Caspase-3 (green); scale bar: $10 \mu \mathrm{m}$. (c) Histogram of the proportion of activated Caspase- $3^{+}$cells among Krm1-expressing cells upon co-transfection with increasing amounts of GFP or various Krm1 ligands and normalized to 1 (mean \pm S.D.); ${ }^{*} P<0.001$ and ${ }^{\S} P<0.01$ using Student's $t$-test. (d) Quantification of the activated Caspase-3 fluorescence relative to HA fluorescence per cell measured for $n=139,58,120$ and 63 Caspase- $3^{+}$cells. Each dot represents one cell, log2 scale, ${ }^{*} P<0.001$ using Kolmogorov-Smirnov test. (e) Quantification of the proportion of activated Caspase- $3^{+}$cells among Krm1-HA ${ }^{+}$cells normalized to 1 (mean \pm S.D.) in the absence or presence of $0.1,0.2,0.5$ and $1 \mu \mathrm{g} / \mathrm{ml}$ recombinant Dkk1; ${ }^{*} P<0.001$ using Student's $t$-test. (f) Quantification of the proportion of activated Caspase- $3^{+}$cells among Krm1-HA ${ }^{+}$cells cultured in a medium previously conditioned by GFP- or Dkk1-transfected cells, normalized to 1 (mean \pm S.D.); ${ }^{*} P<0.001$ using Student's $t$-test. (g) Quantification of the proportion of activated Caspase- $3^{+}$cells among Krm1-HA ${ }^{+}$cells seeded on a carpet of GFP or Dkk1-transfected cells. ${ }^{\S} P<0.01$ using Student's $t$-test

decided to further investigate the relationship between Krm1mediated Wnt antagonism and apoptosis promotion.

Consistent with our whole embryo culture experiments, we found that the treatment of Krm1-transfected cells with the Wnt inhibitor endo-IWR1 was unable to recapitulate the antiapoptotic effect of Dkk1 (Figure 4a). In addition, the Wnt activator Azakenpaullone ${ }^{30}$ proved unable to counterbalance Dkk1-mediated rescue of Krm1-induced apoptosis (Figure 4a). Together, these results indicate that the ability of Dkk1 to block apoptotic signaling downstream Krm1 is not mediated by Wnt inhibition. We then performed Wnt-activity assays using the luciferase Wnt reporter TOPFlash. We found that human embryonic kidney (HEK) cells display an intrinsic Wnt activity that was significantly inhibited following $\mathrm{Krm} 1$ expression (Figure 4b). We observed a similar inhibition of Wnt signaling using the Krm1 $\triangle I C D$ construct (Figure $4 b$ ) indicating that the ICD of Krm1 is dispensable for Wnt antagonism, as previously shown for Krm2. ${ }^{17}$ Assessment of the consequence of Krm1 ECD removal on Wnt inhibition proved more difficult to interpret as it appeared highly variable between experiments (Figure 4b). This is perhaps due to the strong apoptotic activity and low expression level of this construct (see Figure 2g). Thus, an apoptotically inactive mutant of Krm1 fully retains its ability to inhibit Wnt signaling, whereas an apoptotically hyperactive form only mediates weak (if any) Wnt antagonism (Figure 4c). We therefore propose a model, in which Krm1 display two independent signaling activities: Wnt inhibition through its ECD in the presence of Dkk1 and apoptosis induction through its cytoplasmic domain in the absence of ligand (Figure 4d).

Krm1 apoptotic activity is a recent evolutionary acquisition. Our data indicate that Krm2 does not share the same intrinsic apoptotic activity as Krm1, we therefore speculated that such an activity was acquired by Krm1 (or lost by Krm2) during evolution. To test our hypothesis and determine when the pro-apoptotic function might have arisen, we transfected cells with plasmids encoding chicken, xenopus and zebrafish Krm1. Incidentally, only one Krm1 ortholog was found in each of these species (compared sequences of the ICDs are shown in Figure 5a). Contrary to $\mathrm{mKrm} 1$, we found that cKrm1, xKrm1 and zKrm1 were unable to induce Caspase-3 activation (Figures 5b-e, compare with Figure 2a). This may either result from a lack of intrinsic pro-apoptotic activity of chicken, xenopus and zebrafish Krm1 or from their inability to interact with the necessary partners in human cells. To distinguish between these two possibilities, we tested the ability of $\mathrm{mKrm} 1$ and $\mathrm{cKrm} 1$ to induce apoptosis in chick embryos using in ovo electroporation. As revealed by TUNEL staining, we found that $\mathrm{mKrm} 1$ is able to induce cell death in avian cells (Figures $5 f$ and $h$ ), not only indicating that $\mathrm{mKrm} 1$ is indeed apoptotic in an in vivo context, but also that the 

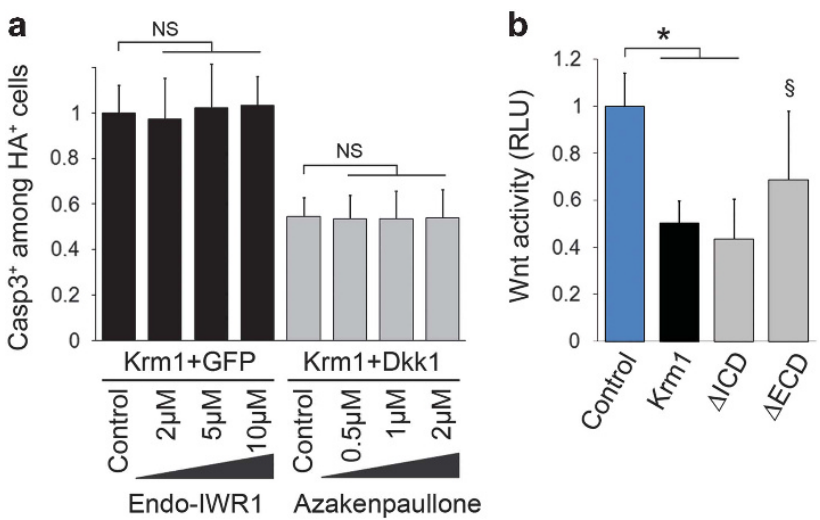

Endo-IWR1 Azakenpaullone

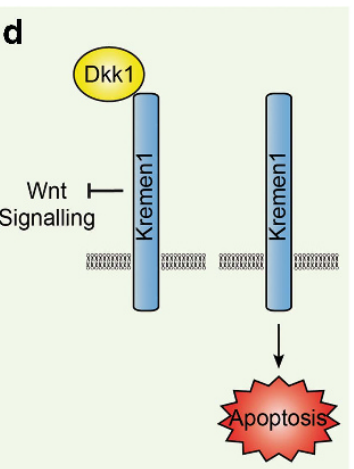

Figure 4 Kremen1 acts in a Wnt-independent manner. (a) Quantification of the proportion of activated Caspase- $3^{+}$cells among $\mathrm{HA}^{+}$cells following co-transfection with either Krm1 and GFP (black bars) or Krm1 and Dkk1 (gray bars). Inhibition of the Wnt pathway using increasing doses of endo-IWR1 does not affect Krm1-induced apoptosis. Activation of the Wnt pathway with increasing doses of Azakenpaullone has no effect on Dkk1-mediated rescue of Krm1-induced apoptosis, NS: nonsignificant. (b) Luciferase assay indicating the relative activity of the Wntsignaling pathway in cells transfected with Krm1 or Krm1 truncations, compared with control cells; mean \pm S.D., ${ }^{\star} P<0.001$ using Student's $t$-test, ${ }^{\S} P<0.05$ compared with control or with Krm1 using Student's t-test. (c) Summary of apoptotic and antiWnt activities of $\mathrm{Krm} 1$ and its truncation mutants as shown in Figures $2 \mathrm{~h}$ and $4 \mathrm{~b}$. (d) A model summarizing our findings: Krm1 mediates Wnt inhibition in the presence of Dkk1 and triggers apoptosis in its absence

downstream signaling pathway most likely involves partners that are conserved between chick and mouse. Unlike its murine counterpart, cKrm1 was unable to trigger apoptosis in ovo (Figures $5 \mathrm{~g}$ and $\mathrm{h}$ ), further supporting the hypothesis of an evolutionary acquisition of a pro-apoptotic behavior by $\mathrm{mKrm} 1$.

We then aimed at identifying residues or motifs within the ICD of mKrm1 that permit apoptotic signaling to occur. We first made the fortuitous observation that the addition of an epitope tag to the $\mathrm{C}$ terminus of $\mathrm{mKrm} 1$ completely abolishes Caspase-3 activation (Figure 6a). We therefore speculated that the C-terminal domain of $\mathrm{mKrm} 1$ is essential for its proapoptotic function. Sequence comparison between mouse, chicken, xenopus and zebrafish Krm1 ICDs prompted us to focus on the last two residues of $\mathrm{mKrm} 1$, which are the only ones in the last dozen not to be found in any of the three other species (Figure 5a). We found that, in $\mathrm{mKrm1}$, substitution of either one of the last two amino acids by their chicken counterpart (S472G or D473N) completely abolished Caspase-3 activation (Figure 6a). Conversely, in cKrm1, substitution of the last two residues (GN) with their murine equivalent (S.D.) was sufficient to confer apoptotic activity, although not to a full extent, to the normally inactive chick protein (Figures $6 \mathrm{~b}-\mathrm{e}$ ). We therefore concluded that $\mathrm{C}$-terminal residues of $\mathrm{mKrm} 1$ are critical for triggering apoptosis.

Consistent with our previous observation that Wnt antagonism is mediated by the ECD, we found that the various mouse and chicken Krm1 constructs, which differ in their apoptotic activities, share the same ability to inhibit Wnt signaling (Figure 6f), further confirming that Krm1-induced apoptosis is Wnt independent.

Almost all Krm1 sequences of placental mammals we found in the databases show strong conservation at the $\mathrm{C}$ terminus (including S.D. residues), whereas none of the amphibians, sauropsids (birds, crocodiles and turtles) or bony fishes have the S.D. motif (Figure 6g). Moreover, among nonplacental mammals, platypus has GY residues at the $C$ terminus, whereas the marsupials opossum and wallaby display a GD motif; sequences which are, according to our mutagenesis experiments, unlikely to allow apoptotic signaling in these three species. We therefore propose that the pro-apoptotic activity of $\mathrm{Krm} 1$ was acquired subsequent to the divergence between marsupials and placental mammals. Furthermore, in humans (as well as several primates) alternative splicing can yield an additional isoform that differs at the $\mathrm{C}$ terminus, consisting in the replacement of the last three amino acids by a 20 amino-acids-long tail (Figure 6g). We speculated that this 'long' isoform would be apoptotically inactive and tested this hypothesis by substituting the last three amino acids of $\mathrm{mKrm} 1$ by the 20 amino-acid tail. As illustrated in Figure 6h, we found the long isoform unable to trigger apoptosis, further demonstrating the importance of the $\mathrm{C}$-terminal sequence.

Krm1 apoptotic activity is reduced by cancer mutations. Dependence receptors have been proposed to act as tumor suppressors and improper function of dependence receptors has been reported in cancers. ${ }^{1}$

Consistent with the involvement of $\mathrm{Krm} 1$ in human cancers we found in the cancer genome atlas (TCGA; https://genomecancer.ucsc.edu) ${ }^{31}$ that various cancers fit within a pattern combining (i) high expression of $\mathrm{Krm} 1$ and low expression of Dkk1 in normal tissue, (ii) decreased Krm1 expression in tumors, and/or (iii) increased Dkk1 expression in tumors (Supplementary Figure 3). To further question the contribution of Krm1-induced apoptosis in cancers, we interrogated the catalogue of somatic mutations in cancer database (COSMIC; http://www.sanger.ac.uk/cosmic). ${ }^{32}$ At the date we performed our studies (COSMIC v68 release, 4 February 2014), 45 unique samples bearing $\mathrm{krm} 1$ mutations were reported (out of 8845), with some of these mutations being found in several independent samples. By contrast, only 12 mutations in $\mathrm{krm} 2$ were found, all of them in unique samples and nearly half of them being coding silent. We decided to focus on the three missense point mutations identified within Krm1 ICD: S421F (found in two colorectal and one ovary carcinoma samples), S439L (found in one lung carcinoma sample) and I455V (also found in one lung carcinoma sample). It is worth noting that a second sample (colorectal carcinoma) bearing the S439L mutation was added to the COSMIC database in a more recent release ( $v 70$ release, 14 August 2014). 


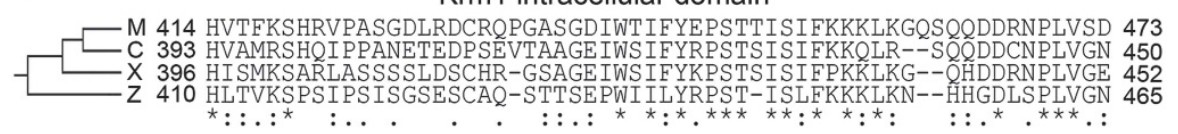

DAPI GFP Activated Caspase-3
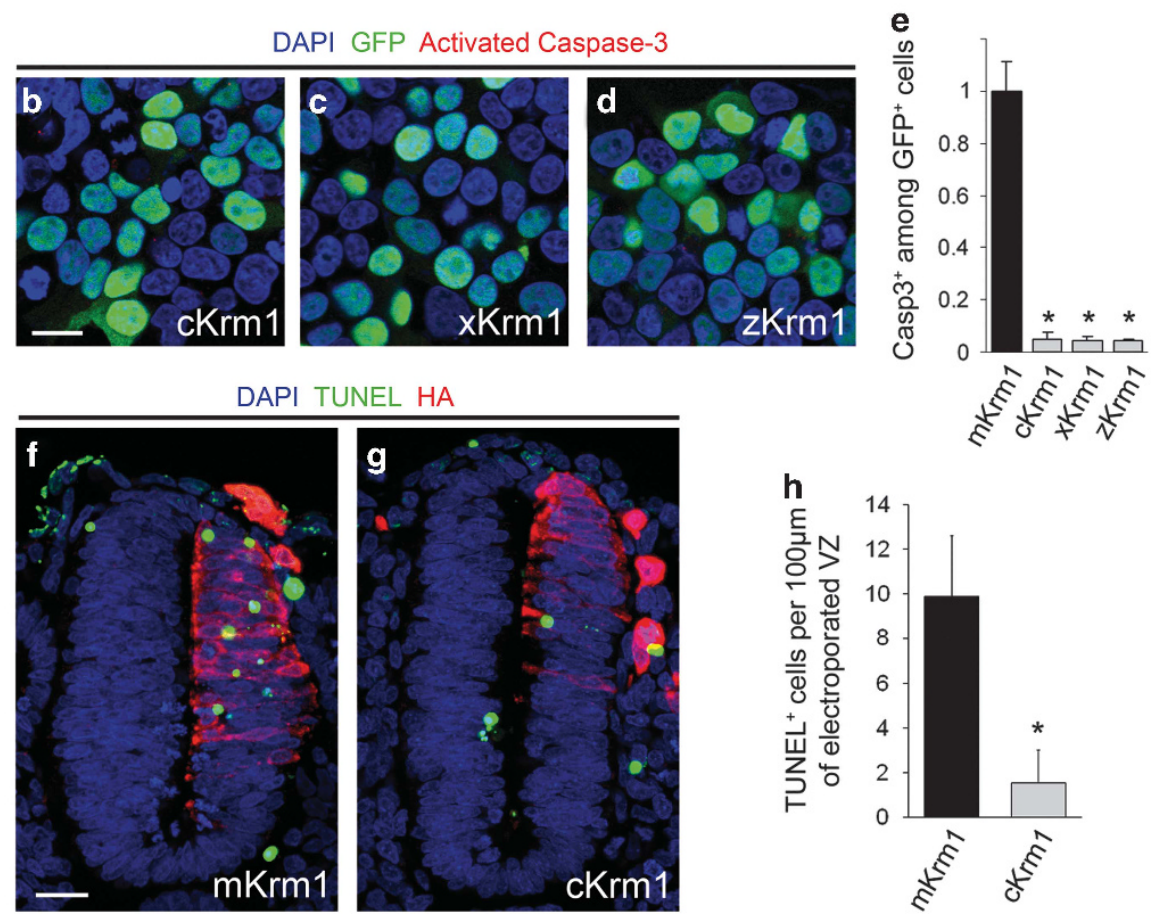

Figure 5 Kremen1 apoptotic activity is not common among vertebrates. (a) Protein-sequence comparison of the intracellular domain of Krm1 in mouse (amino acids 414-473), chick (393-450), xenopus (396-452) and zebrafish (410-465). Conservation of residues is indicated below according to the nomenclature of the ClustalX2 software. (b-d) HEK293T cells transfected with cKrm1 (b), xKrm1 (c), or zKrm1 (d) are identified by GFP expression (green). Activated Caspase-3 immunostaining (in red) is negative in all three conditions; scale bar in (b): $10 \mu \mathrm{m}$. (e) Histogram representing the proportion of activated Caspase- $3^{+}$cells among GFP ${ }^{+}$cells in the experiments shown in (b-d) and normalized to 1 (mean \pm S.D.); ${ }^{*} P<0.001$ using Student's $t$-test. (f and g) Cryosections of chick spinal cord electroporated with HA-tagged mKrm1 (f) or cKrm1 (g). Transfected cells are shown in red (HA immunolabeling) and apoptotic cells in green (TUNEL staining); scale bar in (f): $20 \mu \mathrm{m}$. (h) Histogram representing the number of apoptotic cells counted per $100 \mu \mathrm{m}$ of electroporated ventricular zone in the experiments shown in (f and $\mathbf{g})$ (mean \pm S.D.); ${ }^{*} P<0.001$ using Student's $t$-test

To address whether these mutations affect Krm1 proapoptotic activity, we mutated the corresponding amino acids of mouse Krm1 (S419F, S437L and I453V) and proceeded with cell transfection (Figures $7 a-d$ ). We found that transfection with S419F or S437L mutants induced Caspase-3 activation in $\sim 40 \%$ less cells than their wild-type counterpart (Figure 7e). In addition, we measured the intensity of activated Caspase-3 staining relative to HA staining per cell and found that all three mutations resulted in a significant reduction (Figure 7f). Importantly, and consistent with our previous finding, Krm1 cancer mutants appear equally able to antagonize Wnt signaling as wild-type Krm1 (Figure 7g). We therefore concluded that Krm1 mutations found in human cancers can affect its apoptotic activity and propose that these mutations favor abnormal cancer cells survival and thus confer them a selective advantage (Figure $7 \mathrm{~h}$ ).

\section{Discussion}

We have unraveled an unexpected function for Dkk1 and Krm1 in regulating cell survival, in addition to (and independent of) their previously characterized role in Wnt signaling. To our knowledge, it is the first demonstration of a Wnt-independent function for both Dkk1 and Krm1, and the first report of a biological function that is not shared by $\mathrm{Krm} 1$ and $\mathrm{Krm} 2$ as both were shown to equally mediate Dkk1-induced Wnt inhibition. ${ }^{17}$

A Krm2 truncation mutant lacking the entire ICD was previously shown to retain its ability to mediate Wnt inhibition. ${ }^{17}$ Consistently, we found that Krm1 ICD is also dispensable for Wnt inhibition. Conversely, the cytoplasmic tail of $\mathrm{Krm} 1$ is strictly required for apoptotic signaling. Krm1 therefore appears as an atypical dependence receptor as its positive and negative signaling activities are regulated by topologically distinct domains.

In this study, we report for the first time a specific function for mouse Krm1 compared with its chick, xenopus and zebrafish orthologues. Interestingly, our cross-species electroporation experiments indicated that $\mathrm{mKrm} 1$ retains its apoptotic activity when expressed in avian cells. It is therefore reasonable to assume that the intracellular apoptotic signaling cascade was already present in the common ancestor to placental mammals. We can thus speculate that the acquisition of a pro-apoptotic behavior by Krm1 occurred by a mechanism of molecular exaptation, consisting in the recruitment of a preexisting functional pathway. 

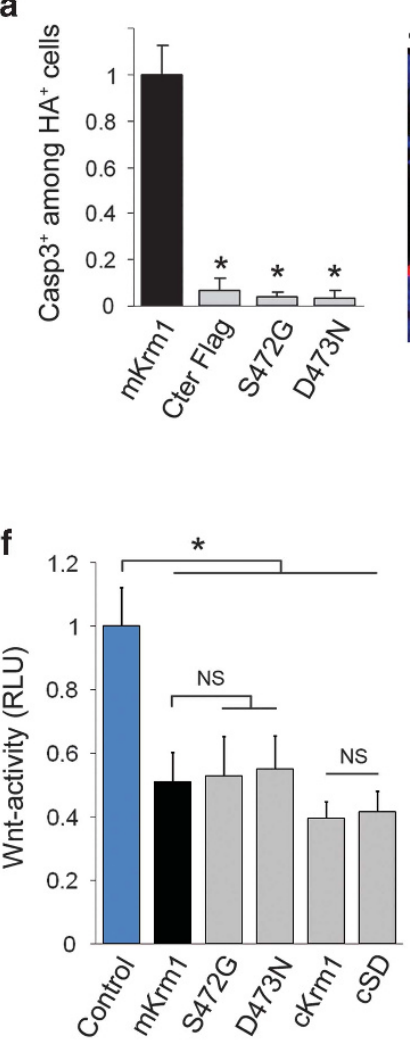

DAPI Activated Caspase-3 HA
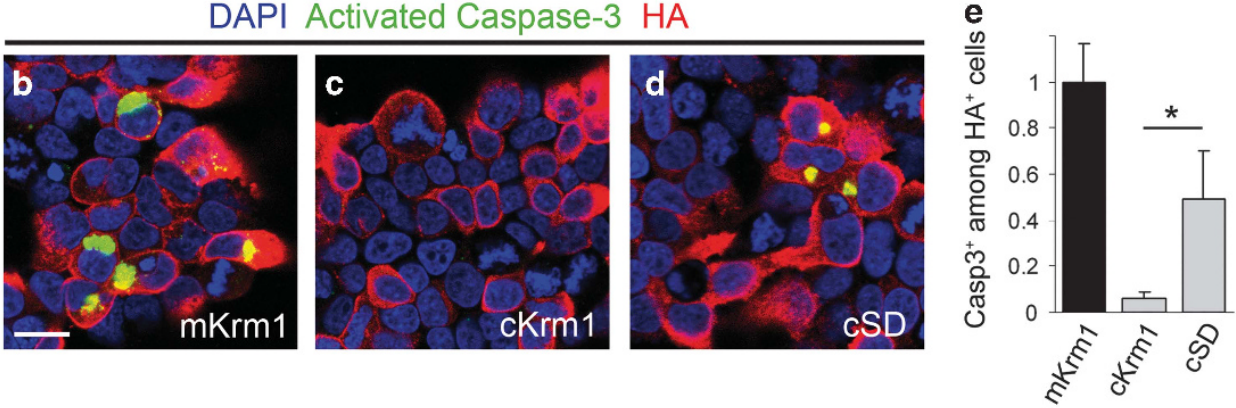

g

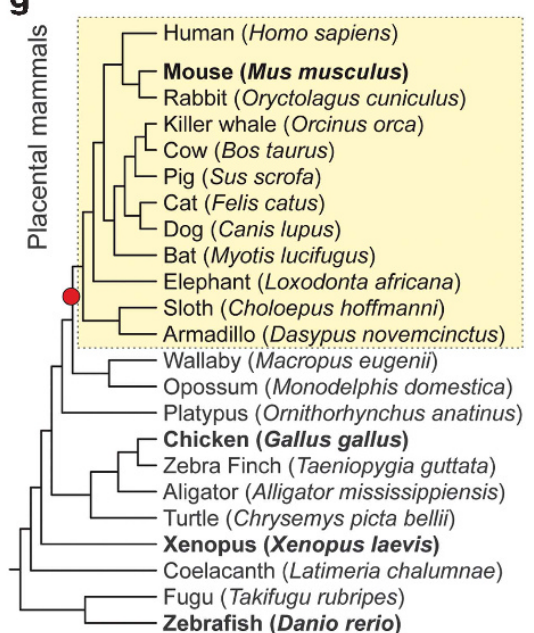

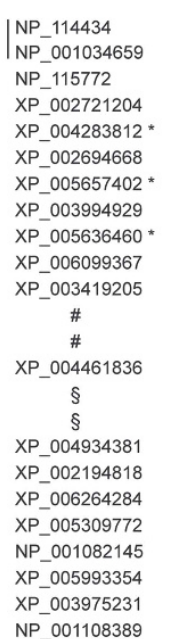
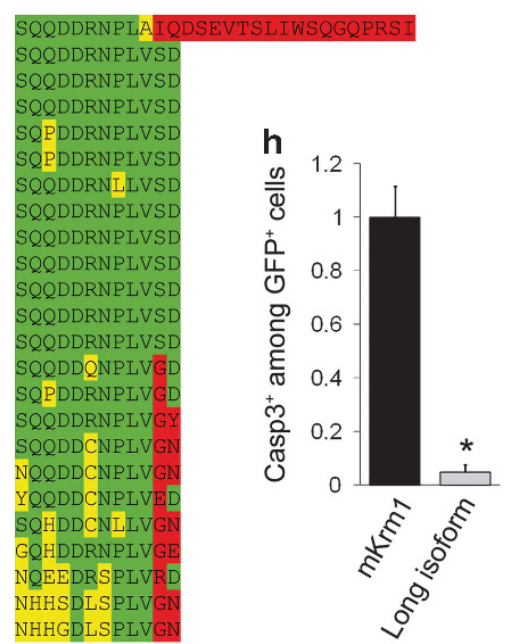

Figure 6 Kremen1 C-terminal domain is responsible for apoptotic signaling. (a) Quantification of the proportion of activated Caspase- $3^{+}$cells among $\mathrm{HA}^{+}$cells following transfection of $\mathrm{mKrm1}$, Krm1 bearing a C-terminal Flag tag or point mutants S472G and D473N, normalized to 1 (mean \pm S.D.); ${ }^{*} P<0.001$ using Student's $t$-test. (b-d) HEK293T cells transfected with HA-tagged mKrm1 (b), cKrm1 (c), or a modified cKrm1 bearing $S$ and $D$ residues at the $C$ terminus (d). HA $A^{+}$-transfected cells are shown in red and activated Caspase- $3^{+}$cells in green; scale bar in (b): $10 \mu \mathrm{m}$. (e) Quantification of the proportion of activated Caspase- $3^{+}$cells among HA ${ }^{+}$cells in the experiments shown in (b-d) normalized to 1 (mean \pm S.D.); ${ }^{*} P<0.001$ using Student's $t$-test. (f) Luciferase assay indicating the relative activity of the Wnt-signaling pathway (mean \pm S.D.) in cells transfected with wild type or mutant mouse and chicken Krm1 constructs; ${ }^{*} P<0.001$ using Student's t-test. NS: nonsignificant. (g) Phylogenetic tree of various vertebrates for which a krm1 sequence was found. Length of the branches is arbitrary. Species in bold are those for which we tested Krm1 apoptotic activity. RefSeq accession numbers are given when available. The two human sequences are generated by alternative splicing. *indicate cases where several sequences were found but with identical $\mathrm{C}$ termini. " $\mathrm{Sloth}$ and armadillo sequences were manually reconstructed using Ensembl. ${ }^{\S}$ Opossum and platypus sequences are referenced in UniProtKB under the accession numbers $\mathrm{H} 9 \mathrm{H} 6 \mathrm{Q} 3$ and F7FX81, respectively. Protein sequences of the $\mathrm{C}$ termini were aligned. Residues identical to the mouse sequence are highlighted in green. Residues that differ from the mouse sequence are indicated in red when located at positions that are critical for Krm1 apoptotic activity and in yellow otherwise. The red circle on the phylogenetic tree represents the stage at which the apoptotic activity of Krm1 was most likely acquired (lineage of the placental mammals). (h) Histogram representing the proportion of activated Caspase- $3^{+}$cells among $\mathrm{GFP}^{+}$cells following transfection of $\mathrm{mKrm} 1$ or a modified version bearing the same $\mathrm{C}$ terminus as the human long isoform, normalized to 1 (mean \pm S.D.); ${ }^{*} P<0.001$ using Student's t-test

Loss of function experiments in xenopus demonstrated that Krm1 and Krm2 are required together for amphibian anterior nervous system development. ${ }^{24}$ In mice, however, $\mathrm{Krm} 1^{-/-}$; $\mathrm{Krm2}^{-/}$animals are viable and display no obvious brain phenotype. ${ }^{33}$ In these two species, requirement of Kremens during central nervous system (CNS) development and apoptotic activity of $\mathrm{Krm} 1$ therefore appears inversely correlated. One possibility is that the acquisition of apoptotic behavior by $\mathrm{Krm} 1$ is simply not compatible with a strong requirement during development. Another possibility is that the acquisition of apoptotic activity by Krm1 was accompanied by inhibitory mechanisms preventing deleterious functioning. Our expression data, consistent with other studies ${ }^{18,19,24,34,35}$ indicate that $\mathrm{krm} 1$ is a rather widely expressed gene during mouse embryonic development, whereas $d k k 1$ expression is more spatially restricted. This may well reflect the existence of mechanisms negatively regulating Krm1-induced apoptosis, and whose identification will be one of the future challenges.

Cell death has not been investigated in the developing CNS of either $\mathrm{Krm} 1^{-1-}$ or $D k k 1^{-1-}$ embryos, ${ }^{19,33}$ although one can argue that in the case of Dkk1 mutants, the dramatic absence of induction of head structures does not allow such an analysis to be performed. Interestingly, in the hippocampus of adult mice, conditional tamoxifen-induced loss of Dkk1 was shown to result in an increased apoptosis that specifically affects $\mathrm{DCX}^{+}$neuroblasts, ${ }^{36}$ supporting the hypothesis that Dkk1 can act as a survival factor in physiological conditions.

Most dependence receptors identified so far were involved in cancers, consistent with the idea that both receptor loss of function or ligand overexpression may confer a selective advantage to tumor cells by favoring their abnormal survival. ${ }^{1}$ In the specific case of DCC, possibly the most studied 
DAPI Activated Caspase-3 HA

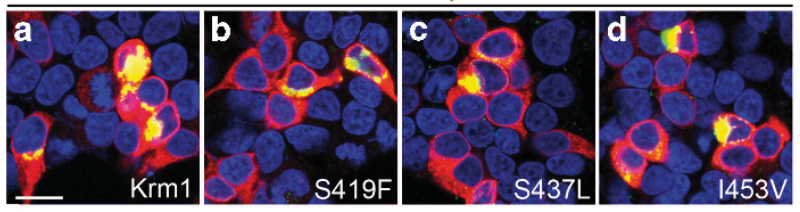

e

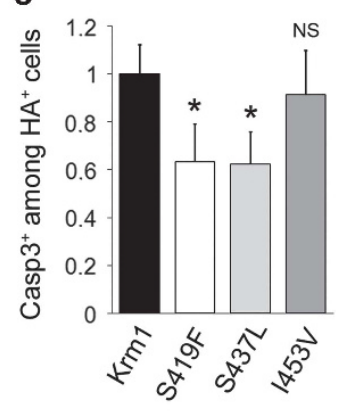

g

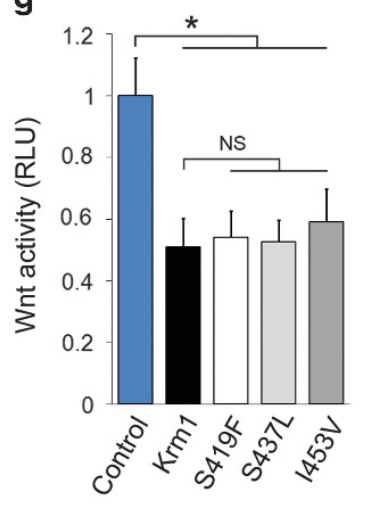

$f$

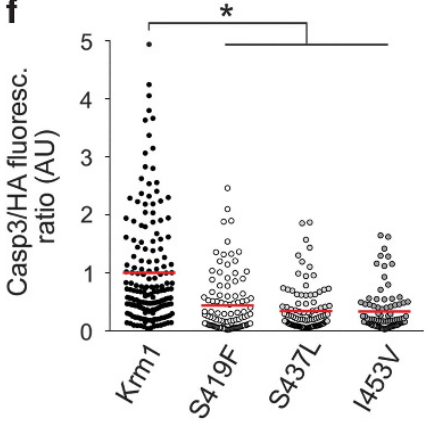

h

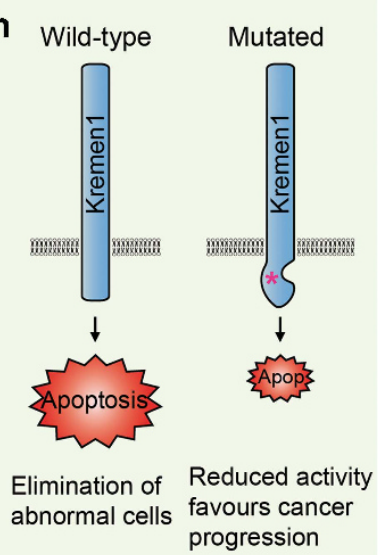

Figure 7 Somatic mutations found in human cancers can affect Krm1 proapoptotic activity. (a-d) HEK293T cells expressing HA-tagged mKrm1 (a) or cancer mutants S419F (b) S437L (c) and I453V (d). Immunostaining revealed $\mathrm{HA}^{+}$cells in red and activated Caspase-3 in green; scale bar in (a): $10 \mu \mathrm{m}$. (e) Quantifications the proportion of activated Caspase-3-positive cells among HA-positive cells in the experiments shown in (a-d), normalized to 1 (mean \pm S.D.); ${ }^{*} P<0.001$ using Student's t-test; NS: nonsignificant. (f) Quantification of the activated Caspase-3 fluorescence relative to HA fluorescence per cell measured for $n=171$ (Krm1), 107 (S419F), 110 (S437L) and 89 (I453V) cells. Each dot represent one cell; ${ }^{*} P<0.001$ using Kolmogorov-Smirnov test. (g) Luciferase assay indicating the relative activity of the Wnt-signaling pathway in cells transfected with wild-type Krm1 or cancer mutants; ${ }^{*} P<0.001$ using Student's $t$-test; NS: nonsignificant. (h) Proposed model: Krm1 apoptotic activity favors the elimination of abnormal cells; mutations affecting its ability to activate Caspase-3 may confer a selective advantage to cancer cells

dependence receptor, the tumor suppressor function has formally been demonstrated and attributed to its apoptotic activity. ${ }^{37}$ DCc mutations are found in $\sim 2.5 \%$ of COSMIC samples compared with $\sim 0.5 \%$ for $\mathrm{krm} 1$. However, since dcc is a gene three times longer than $\mathrm{krm} 1$, and therefore more likely to accumulate mutations, the frequency of $\mathrm{krm} 1$ mutations in cancers appears comparable to that of $d c c$.

Identifying new dependence receptors remains of primary importance in the scope of providing alternative cancer therapies and diagnostic tools. New perspectives are currently arising, consisting in characterizing tumors that express both a dependence receptor and its ligand and trying to interfere with production of the latter. ${ }^{38-41}$ We provide strong evidences supporting an evolutionary acquisition of dependence behavior for Krm1, which might be one of the mechanisms by which placental mammals tackled the necessity to develop protective strategies against cancers. We further suggest that in humans, Krm1 apoptotic behavior can be regulated by alternative splicing. Before this study, Krm1 implication in cancers was rather lightly documented, only supported by the loss of expression observed in some cancer cell lines. ${ }^{35} \mathrm{We}$ now provide additional evidence by reporting that somatic mutations in cancer can affect Krm1 apoptotic activity without modifying its ability to inhibit Wnt signaling. Although the cancer mutations we tested are not complete loss of apoptotic function, it is worth mentioning that such a possibility has been reported in at least two instances in the COSMIC database, corresponding to frameshift mutations at positions 251 and 438 that certainly abolish $\mathrm{Krm} 1$ apoptotic activity.

By contrast, the role of Dkk1 in cancers is already well established. Interestingly, despite the fact that all studies concur that Dkk1 acts through modulation of Wnt signaling, both positive and negative roles have been proposed. ${ }^{20-22}$ Such a discrepancy could reflect Dkk1's dual function: as a Wnt antagonist, Dkk1, would inhibit cancer progression, whereas the anti-apoptotic function we reveal would favor cancer cells survival. It is noteworthy that multiple studies provided proof of concept that Dkk1 targeting by immunotherapy can be used efficiently in both protective and therapeutic manners against cancer. $^{42-47}$ Sato et al. ${ }^{46}$ found that anti-Dkk1 treatment was able to induce apoptosis of A549 lung cancer cells (which express high levels of Dkk1) by an unknown mechanism, which we believe could be the one we describe here. Furthermore, tumors formed in nude mice following A549 cells injection were significantly smaller in animals treated with anti-Dkk1 compared with controls, demonstrating that Dkk1 targeting by antibodies can efficiently inhibit tumor growth in vivo. ${ }^{46}$ Our work will therefore not only shed a new light on Dkk1 and Krm1 function in development and cancer, but also (and more significantly) support efforts in developing therapies based on interfering with this novel pathway.

\section{Materials and Methods}

Animals. All animals used in this study were handled according to national regulations (approval \#5096 from the French Ministry of Research on the use of genetically modified animals) and approved by the Veterinary Services of Paris (authorization to perform experiments on vertebrate animals \#75-1454). Ablated embryos were obtained by mating PGK:Cre and Dbx $1^{\text {LoxP-Stop-LoxP-DTA }}$ animals as previously reported. $^{23}$

Constructs. The coding sequences of mouse krm1, krm2, dkk1, dkk2, dkk3, $d k k 4$ and rspo1 were obtained following RNA extraction of E12.5 embryos using Trizol (Invitrogen, Carlsbad, CA, USA), cDNA synthesis using SuperScript VILO (Invitrogen) and PCR amplification using Phusion polymerase (Thermo Scientific, Waltham, MA, USA). Human Irp6 was amplified from I.M.A.G.E. clone \#40125687. Chick, xenopus and zebrafish krm1 were obtained from cDNA of HH14, stage 20 and $22 \mathrm{hpf}$ embryos, respectively. Constructs were made in pCAG-IRES-NLS-EGFP, mKrm1 was also cloned in pCS2. In some instances, an HA tag was inserted after the signal peptide. In the specific case of $\mathrm{mKrm} 1$ in pCS2, we found that adding the HA tag before the signal peptide had no incidence on expression levels, surface localization or apoptotic activity. For reasons that remain unclear, a better correlation between HA and Caspase-3 fluorescence intensities was observed with such a construct. Point mutations were generated using QuickChange site-directed 
mutagenesis kit (Stratagene, La Jolla, CA, USA), truncation or deletion mutants were produced by fusion PCR.

Culture. Whole embryo culture was performed in $75 \%$ rat serum (Charles River, Japan) in DMEM supplemented with penicillin and streptomycin using a $5 \% \mathrm{CO}_{2}$ incubator.

For immunofluorescence experiments, HEK293T cells were seeded on poly-Llysine-coated glass coverslips (in $15 \mathrm{~mm}$ wells) and cultured in 10\% fetal bovine serum in DMEM supplemented with penicillin and streptomycin. Transfection was performed for $4 \mathrm{~h}$ in Optimem using $2 \mu \mathrm{l}$ Lipofectamine 2000 (Invitrogen) and $1 \mu \mathrm{g}$

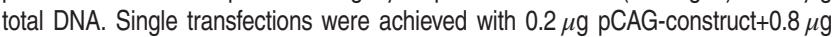
empty pBluescript. Co-transfections were done using pCS2-Krm1 and pCAG-ligand in respective ratios of $0.5 \mu \mathrm{g} / 0.5 \mu \mathrm{g}$ (Figures $3 \mathrm{a}-\mathrm{d}$ and $4 \mathrm{a}$ ) or $0.2 \mu \mathrm{g} / 0.8 \mu \mathrm{g}$ (Figures $3 \mathrm{c}$ and d). Carpets of $\mathrm{GFP}^{+}$or $\mathrm{Dkk}^{+}$cells were obtained by transfecting $1 \mu \mathrm{g} \mathrm{pCAG-GFP}$ or pCAG-Dkk1 4-6 $\mathrm{h}$ before seeding of dissociated $\mathrm{Krm} 1^{+}$cells. Conditioned media were collected $24 \mathrm{~h}$ after transfection with $1 \mu \mathrm{g}$ pCAG-GFP or pCAG-Dkk1.

For Wnt-activity assays, HEK293T cells were seeded on 96-well plates and transfected with $10 \mathrm{ng}$ pRL-Renilla, $10 \mathrm{ng}$ TOPFlash and $40 \mathrm{ng}$ pCAG-Krm1 constructs (completed to $200 \mathrm{ng}$ total DNA with empty pBluescript) using $0.4 \mu \mathrm{l}$ Lipofectamine 2000 per well.

Recombinant mouse Dkk1 (R\&D systems) was used at concentrations ranging from 0.1 to $1 \mu \mathrm{g} / \mathrm{ml}$. We found the protein most efficient when used shortly after resuspension and therefore used only recently prepared aliquots. The Axin2 stabilizer Endo-IWR1 (Tocris, Minneapolis, MN, USA) was used at concentrations ranging from 2 to $10 \mu \mathrm{M}$ to inhibit Wnt signaling, ${ }^{25}$ the GSK-3 $\beta$ inhibitor 1-Azakenpaullone (SigmaAldrich, St. Louis, MO, USA) was used at concentrations ranging from 0.5 to $2 \mu \mathrm{M}$ to activate Wnt signaling. ${ }^{30}$

Staining. In situ hybridization and TUNEL were performed as previously described. ${ }^{23}$ Immunostaining was performed $24 \mathrm{~h}$ after cell transfection, the following antibodies were used: mouse anti-HA 16B12 (Convance, Princeton, NJ, USA; 1: 1000), rabbit anti-cleaved Caspase-3 (Cell Signaling Technology, Beverly, MA, USA; 1:1000), chick anti-GFP (Aves Labs, Tigard, OR, USA; 1:2000). Secondary antibodies were coupled to Alexa488 (Invitrogen), Cy3 or Cy5 (Jackson Immunoresearch, West Grove, PA, USA).

Wnt-activity assay. Twenty-four hours after transfection, cells were rinsed in PBS and lysed in PLB buffer (Promega, Madison, WI, USA) for $25 \mathrm{~min}$ at room temperature. Firefly and Renilla luciferase activities were measured sequentially using the Dual Luciferase Reporter Assay kit (Promega) and a TriStar LB941 plate reader (Berthold Technologies, Bad Wildbad, Germany).

Chick electroporation. Fertilized chick eggs were obtained from Morizeau (Dangers, France) and incubated at $37^{\circ} \mathrm{C}$ until HH10. Following DNA $(4 \mu \mathrm{g} / \mu \mathrm{l})$ injection in the spinal cord, five pulses of $25 \mathrm{~V}$ were applied using a CUY21 electroporator and CUY611P7-2 electrodes (Nepagene, Chiba, Japan) to achieve unilateral transfection. Embryos were collected $24 \mathrm{~h}$ later.

Quantifications. Quantification of apoptosis in cultured embryos was achieved by counting the number of TUNEL ${ }^{+}$cells in the neural plate on $20 \mu$ m-thick sections obtained from 3 to 5 embryos per condition. Means were compared using Student's $t$-test. Distributions were also compared by pooling all sections $(n=25-36$ depending on the condition) and applying the nonparametric Kolmogorov-Smirnov test. Quantification of apoptosis in HEK cells was achieved by counting the proportion of active Caspase- $3^{+}$cells among $\mathrm{GFP}^{+}$(for untagged constructs) or $\mathrm{HA}^{+}$ cells. Experiments were performed at least in triplicates. Approximately $200 \mathrm{GFP}^{+}$or $\mathrm{HA}^{+}$cells were considered for each experiment. Histograms represent the means normalized to 1 in control condition (usually Krm1 alone). Error bars on histograms correspond to standard deviations. Means were compared using Student's $t$-test. The activated Caspase-3 fluorescence relative to HA fluorescence was calculated for individual cells (the number of cells considered is indicated in the figure legends) and normalized to 1 in control condition. Distributions were compared using Kolmogorov-Smirnov test. Quantifications of Wnt-signaling activities in HEK cells were achieved by dividing the Firefly luciferase by Renilla in each well, and is expressed in relative luminescence units normalized to 1 in control condition (untreated HEK cells). Experiments were performed at least in quadruplicates. Quantification of apoptosis in electroporated chick embryos was achieved by counting the number of TUNEL ${ }^{+}$cells per $100 \mu \mathrm{m}$ of electroporated ventricular zone from a minimum of six sections belonging to at least three animals for each condition.

\section{Conflict of Interest}

The authors declare no conflict of interest.

Acknowledgements. We are grateful to Thierry Galli, Anne Camus, Stéphane Nedelec, Eva Coppola and Sonia Garel for helpful discussions and critical reading of the manuscript. We wish to thank Anne Camus and Yoko Arai for their help in implementing whole embryo cultures, Christine Vesque and Sylvie SchneiderMaunoury for providing zebrafish cDNA, De-Li Shi for providing xenopus CDNA, Thierry Galli for providing HEK cells, Eve Gazave for her help regarding pharmacological agents, Anne-Laure Todeschini for her help with luciferase assays, Fabien Fauchereau for TCGA data mining, Lisa Vigier and Annie Dutriaux for technical assistance, Betty Freret-Hodara for helpful comments, Elenat Marot, Natalia Maties and Sébastien Gravat for maintaining mouse colonies, as well as the ImagoSeine imaging facility (member of the France Biolmaging infrastructure, supported by the French National Research Agency ANR-10-INSB-04 'Investments for the future'). FC is an Inserm researcher. AP is a CNRS investigator and member Team of the Ecole des Neurosciences de Paris (ENP). This work was supported by grants from the Agence Nationale de la Recherche (ANR-2011-BSV4-023-01), the Fondation pour la Recherche Médicale (FRM) (INE20060306503 and Equipe FRM DEQ20130326521), Ville de Paris (2006 ASES 102) and the Association pour la Recherche sur le Cancer (ARC) (SFI 2011 1203674) to AP and Comité de Paris de la Ligue contre le cancer (RS14/75-7 and RS15/75-46) to FC.

1. Goldschneider D, Mehlen P. Dependence receptors: a new paradigm in cell signaling and cancer therapy. Oncogene 2010; 29: 1865-1882.

2. Mehlen P, Rabizadeh S, Snipas SJ, Assa-Munt N, Salvesen GS, Bredesen DE. The DCC gene product induces apoptosis by a mechanism requiring receptor proteolysis. Nature 1998; 395: 801-804.

3. Llambi $F$, Causeret F, Bloch-Gallego E, Mehlen P. Netrin-1 acts as a survival factor via its receptors UNC5H and DCC. EMBO J 2001; 20: 2715-2722.

4. Thibert C, Teillet MA, Lapointe F, Mazelin L, Le Douarin NM, Mehlen P. Inhibition of neuroepithelial patched-induced apoptosis by sonic hedgehog. Science 2003; 301 : 843-846.

5. Tauszig-Delamasure S, Yu LY, Cabrera JR, Bouzas-Rodriguez J, Mermet-Bouvier C, Guix C et al. The TrkC receptor induces apoptosis when the dependence receptor notion meets the neurotrophin paradigm. Proc Natl Acad Sci USA 2007; 104: 13361-13366.

6. Nikoletopoulou V, Lickert H, Frade JM, Rencurel C, Giallonardo P, Zhang L et al. Neurotrophin receptors TrkA and TrkC cause neuronal death whereas TrkB does not. Nature 2010; 467: 59-63.

7. Luchino J, Hocine M, Amoureux MC, Gibert B, Bernet A, Royet A et al. Semaphorin $3 E$ suppresses tumor cell death triggered by the plexin D1 dependence receptor in metastatic breast cancers. Cancer Cell 2013; 24: 673-685.

8. Anastas JN, Moon RT. WNT signalling pathways as therapeutic targets in cancer. Nat Rev Cancer 2013; 13: 11-26.

9. van Amerongen $R$, Nusse $R$. Towards an integrated view of Wnt signaling in development. Development 2009; 136: 3205-3214.

10. Nusse R, Varmus HE. Many tumors induced by the mouse mammary tumor virus contain a provirus integrated in the same region of the host genome. Cell 1982; 31: 99-109.

11. McMahon AP, Bradley A. The Wnt-1 (int-1) proto-oncogene is required for development of a large region of the mouse brain. Cell 1990; 62: 1073-1085.

12. Angers S, Moon RT. Proximal events in Wnt signal transduction. Nat Rev Mol Cell Biol 2009; 10: $468-477$.

13. Cruciat $\mathrm{CM}$, Niehrs $\mathrm{C}$. Secreted and transmembrane wnt inhibitors and activators. Cold Spring Harb Perspect Biol 2013; 5: a015081.

14. Bafico A, Liu G, Yaniv A, Gazit A, Aaronson SA. Novel mechanism of Wnt signalling inhibition mediated by Dickkopf-1 interaction with LRP6/Arrow. Nat Cell Biol 2001; 3: 683-686.

15. Mao B, Wu W, Li Y, Hoppe D, Stannek $P$, Glinka A et al. LDL-receptor-related protein 6 is a receptor for Dickkopf proteins. Nature 2001; 411: 321-325.

16. Semënov MV, Tamai K, Brott BK, Kühl M, Sokol S, He X. Head inducer Dickkopf-1 is a ligand for Wnt coreceptor LRP6. Curr Biol 2001; 11: 951-961.

17. Mao B, Wu W, Davidson G, Marhold J, Li M, Mechler BM et al. Kremen proteins are Dickkopf receptors that regulate Wnt/beta-catenin signalling. Nature 2002; 417: 664-667.

18. Glinka A, Wu W, Delius H, Monaghan AP, Blumenstock C, Niehrs C. Dickkopf-1 is a member of a new family of secreted proteins and functions in head induction. Nature 1998; 391 : 357-362.

19. Mukhopadhyay M, Shtrom S, Rodriguez-Esteban C, Chen L, Tsukui T, Gomer L et al. Dickkopf1 is required for embryonic head induction and limb morphogenesis in the mouse. Dev Cell 2001; 1: 423-434. 
20. Niehrs C. Function and biological roles of the Dickkopf family of Wnt modulators. Oncogene 2006; 25: 7469-7481.

21. Rubin JS, Barshishat-Kupper M, Feroze-Merzoug F, Xi ZF. Secreted WNT antagonists as tumor suppressors: pro and con. Front Biosci 2006; 11: 2093-2105.

22. Menezes ME, Devine DJ, Shevde LA, Samant RS. Dickkopf1: a tumor suppressor or metastasis promoter? Int J Cancer 2012; 130: 1477-1483

23. Causeret F, Ensini M, Teissier A, Kessaris N, Richardson WD, Lucas de Couville T et al. Dbx1-expressing cells are necessary for the survival of the mammalian anterior neural and craniofacial structures. PLoS One 2011; 6: e19367.

24. Davidson G, Mao B, del Barco Barrantes I, Niehrs C. Kremen proteins interact with Dickkopf1 to regulate anteroposterior CNS patterning. Development 2002; 129: 5587-5596.

25. Chen B, Dodge ME, Tang W, Lu J, Ma Z, Fan CW et al. Small molecule-mediated disruption of Wnt-dependent signaling in tissue regeneration and cancer. Nat Chem Biol 2009; 5 100-107.

26. Galluzzi L, Vitale I, Abrams JM, Alnemri ES, Baehrecke EH, Blagosklonny MV et al. Molecular definitions of cell death subroutines: recommendations of the Nomenclature Committee on Cell Death 2012. Cell Death Differ 2012; 19: 107-120.

27. Binnerts ME, Kim KA, Bright JM, Patel SM, Tran K, Zhou M et al. R-Spondin1 regulates Wnt signaling by inhibiting internalization of LRP6. Proc Natl Acad Sci USA 2007; 104 14700-14705.

28. Nakamura RE, Hackam AS. Analysis of Dickkopf3 interactions with Wnt signaling receptors Growth Factors 2010; 28: 232-242.

29. De Langhe SP, Sala FG, Del Moral PM, Fairbanks TJ, Yamada KM, Warburton D et al. Dickkopf-1 (DKK1) reveals that fibronectin is a major target of Wnt signaling in branching morphogenesis of the mouse embryonic lung. Dev Biol 2005; 277: 316-331.

30. Kunick $C$, Lauenroth $\mathrm{K}$, Leost M, Meijer L, Lemcke T. 1-Azakenpaullone is a selective inhibitor of glycogen synthase kinase-3 beta. Bioorg Med Chem Lett 2004; 14: 413-416.

31. Goldman M, Craft B, Swatloski T, Ellrott K, Cline M, Diekhans M et al. The UCSC Cancer Genomics Browser: update 2013. Nucl Acids Res 2012; 41: D949-D954.

32. Forbes SA, Bindal N, Bamford S, Cole C, Kok CY, Beare D et al. COSMIC: mining complete cancer genomes in the Catalogue of Somatic Mutations in Cancer. Nucleic Acids Res 2011; 39: D945-D950.

33. Ellwanger K, Saito H, Clément-Lacroix P, Maltry N, Niedermeyer J, Lee WK et al. Targeted disruption of the Wnt regulator Kremen induces limb defects and high bone density. Mol Cell Biol 2008; 28: 4875-4882.

34. Nakamura T, Aoki S, Kitajima K, Takahashi T, Matsumoto K, Nakamura T. Molecular cloning and characterization of Kremen, a novel kringle-containing transmembrane protein. Biochim Biophys Acta 2001; 1518: 63-72.
35. Nakamura T, Nakamura T, Matsumoto K. The functions and possible significance of Kremen as the gatekeeper of Wnt signalling in development and pathology. J Cell Mol Med 2008; 12 : 391-408.

36. Seib DR, Corsini NS, Ellwanger K, Plaas C, Mateos A, Pitzer C et al. Loss of Dickkopf-1 restores neurogenesis in old age and counteracts cognitive decline. Cell Stem Cell 2013; 12 : 204-214.

37. Castets M, Broutier L, Molin Y, Brevet M, Chazot G, Gadot N et al. DCC constrains tumour progression via its dependence receptor activity. Nature 2011; 482: 534-537.

38. Fitamant J, Guenebeaud C, Coissieux MM, Guix C, Treilleux I, Scoazec JY et al. Netrin-1 expression confers a selective advantage for tumor cell survival in metastatic breast cancer. Proc Natl Acad Sci USA 2008; 105: 4850-4855.

39. Delloye-Bourgeois C, Fitamant J, Paradisi A, Cappellen D, Douc-Rasy S, Raquin MA et al. Netrin-1 acts as a survival factor for aggressive neuroblastoma. J Exp Med 2009; 206 : 833-847.

40. Paradisi A, Maisse C, Coissieux MM, Gadot N, Lépinasse F, Delloye-Bourgeois C et al. Netrin-1 up-regulation in inflammatory bowel diseases is required for colorectal cancer progression. Proc Natl Acad Sci USA 2009; 106: 17146-17151.

41. Paradisi A, Creveaux M, Gibert B, Devailly G, Redoulez E, Neves D et al. Combining chemotherapeutic agents and netrin-1 interference potentiates cancer cell death. $E M B O M O l$ Med 2013; 5: 1821-1834

42. Fulciniti M, Tassone P, Hideshima T, Vallet S, Nanjappa P, Ettenberg SA et al. Anti-DKK1 $\mathrm{mAb}(\mathrm{BHQ880})$ as a potential therapeutic agent for multiple myeloma. Blood 2009; 114: 371-379.

43. Glantschnig H, Hampton RA, Lu P, Zhao JZ, Vitelli S, Huang $L$ et al. Generation and selection of novel fully human monoclonal antibodies that neutralize Dickkopf-1 (DKK1) inhibitory function in vitro and increase bone mass in vivo. J Biol Chem 2010; 285: 40135-40147.

44. Pozzi S, Fulciniti M, Yan H, Vallet S, Eda H, Patel K et al. Scadden, H.M. Kronenberg, and N. Raje. In vivo and in vitro effects of a novel anti-Dkk1 neutralizing antibody in multiple myeloma. Bone 2013; 53: 487-496.

45. Qian J, Zheng Y, Zheng C, Wang L, Qin H, Hong S et al. Active vaccination with Dickkopf-1 induces protective and therapeutic antitumor immunity in murine multiple myeloma. Blood 2012; 119: 161-169.

46. Sato N, Yamabuki T, Takano A, Koinuma J, Aragaki M, Masuda K et al. Wnt inhibitor Dickkopf-1 as a target for passive cancer immunotherapy. Cancer Res 2010; 70: 5326-5336.

47. Yaccoby S, Ling W, Zhan F, Walker R, Barlogie B, Shaughnessy Jr JD. Antibody-based inhibition of DKK1 suppresses tumor-induced bone resorption and multiple myeloma growth in vivo. Blood 2007; 109: 2106-2111.

\section{Supplementary Information accompanies this paper on Cell Death and Differentiation website (http://www.nature.com/cdd)}

